Supporting Information

\title{
Highly Stretchable and Compressible Cellulose Ionic Hydrogels for Flexible Strain Sensors
}

Ruiping Tong, ${ }^{\dagger}$ Guangxue Chen, ${ }^{\dagger}$ Danhong Pan, ${ }^{\dagger}$ Haisong Qi, ${ }^{+}, \mathcal{R}$ Ren'ai Li, ${ }^{\dagger}$ Junfei Tian, ${ }^{\dagger}$

$$
\text { Fachuang Lu, }{ }^{\dagger, \mathcal{S}} \text { and Minghui He* }{ }^{*}, \mathcal{S}
$$

†State Key Laboratory of Pulp and Paper Engineering, South China University of Technology, Guangzhou 510640, China.

§Guangdong Engineering Research Center for Green Fine Chemicals, Guangzhou 510640, China.

*E-mail: heminghui@scut.edu.cn 

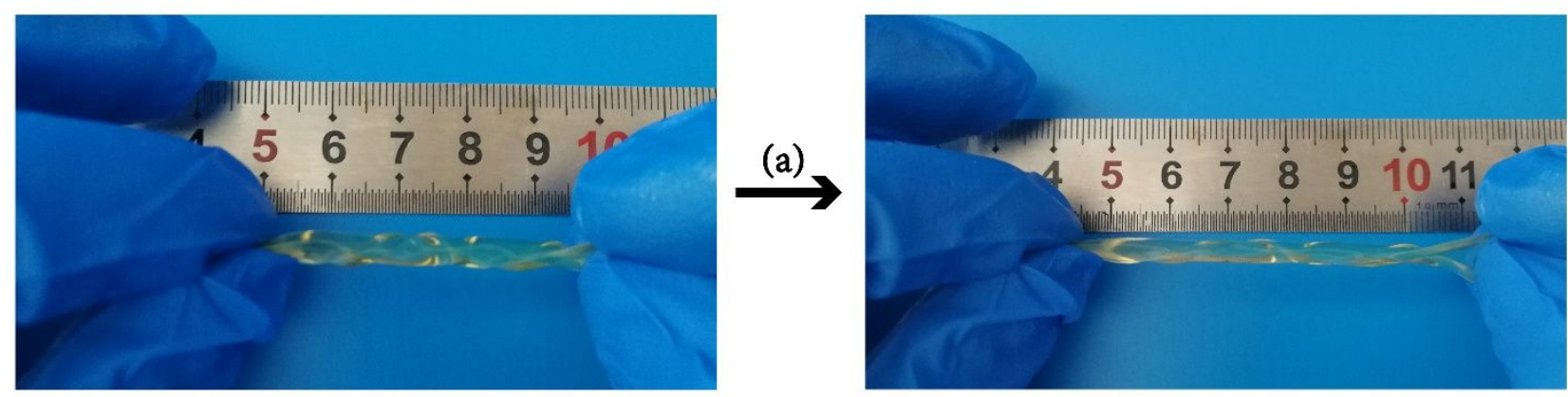

(b)

(c)
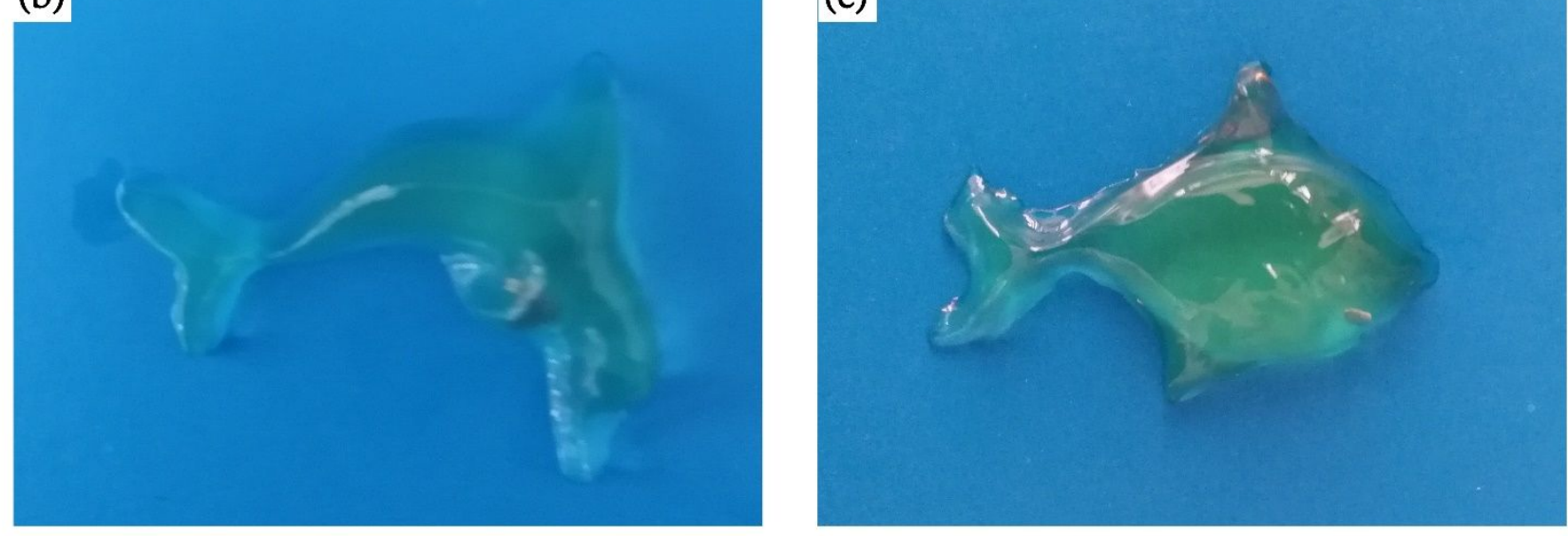

Figure S1. Photographs of cellulose ionic hydrogels (CIHs). (a) Twisted stretching, (b) and (c) free shaping into different fish. 


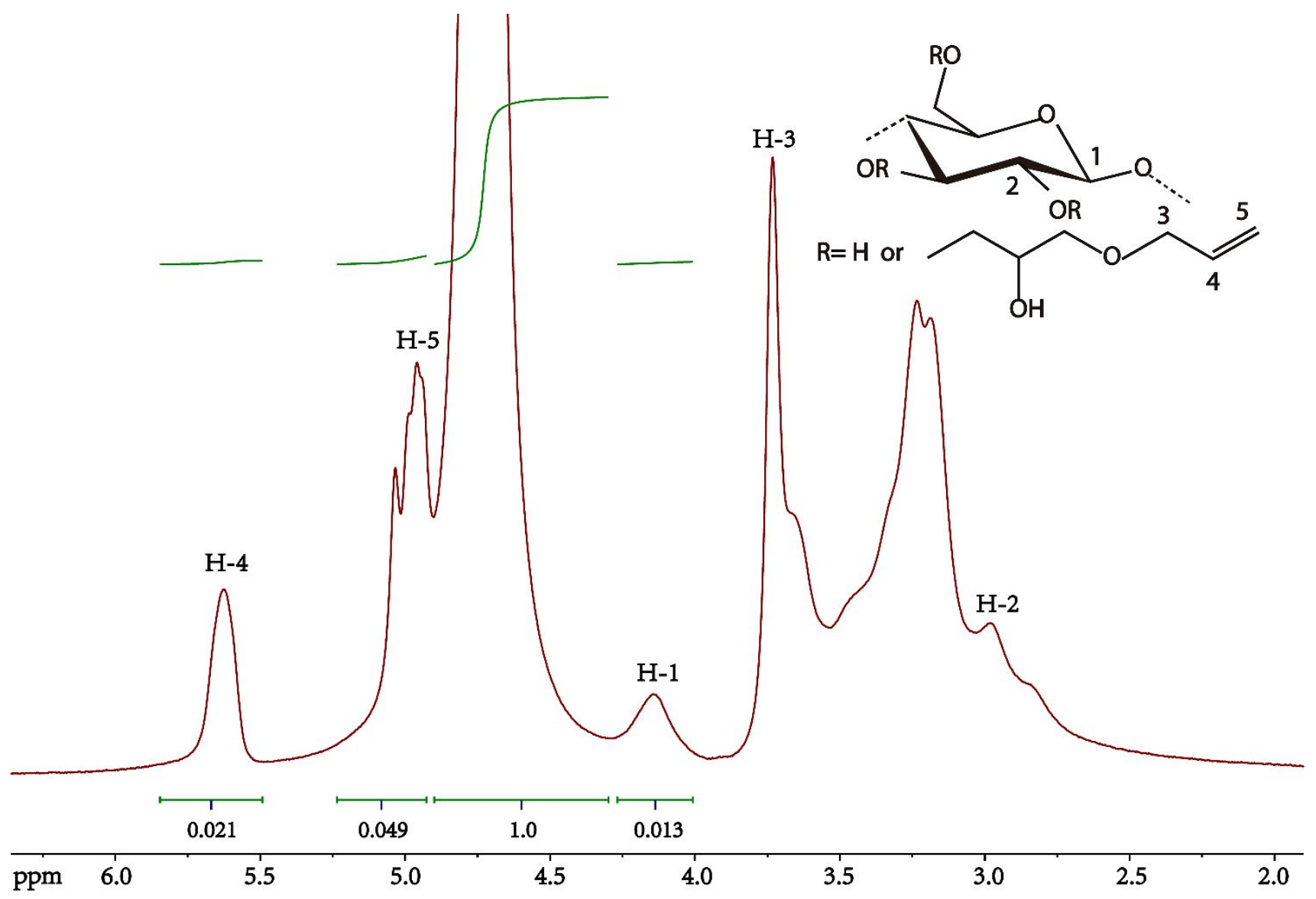

Figure S2. ${ }^{1} \mathrm{H}$ NMR spectra of AC8 (substitution degree $=1.79$ ).

The substitution degree was calculated as follows: ${ }^{1}$

$$
\text { Substitution degree }=\frac{\left(I_{\mathrm{H}-4}+I_{\mathrm{H}-5}\right) / 3}{I_{\mathrm{H}-1}}
$$

Table S1. Conditions and results of synthesis of allyl cellulose (AC) in $7 \mathrm{wt} \% \mathrm{NaOH} / 12 \mathrm{wt} \%$ urea aqueous solution (reaction time $24 \mathrm{~h}$ at $30^{\circ} \mathrm{C}$ )

\begin{tabular}{ccc}
\hline Samples & Molar ratio $^{\text {a) }}$ & Substitution degree $^{\text {b) }}$ \\
\hline AC5 & 5 & 0.75 \\
AC6 & 6 & 1.05 \\
AC7 & 7 & 1.52 \\
AC8 & 8 & 1.79 \\
AC9 & 9 & 2.23 \\
\hline
\end{tabular}

a) Allyl glycidyl ether to anhydroglucose unit (AGU); b) substitution degree determined by ${ }^{1} \mathrm{H}$ NMR. 


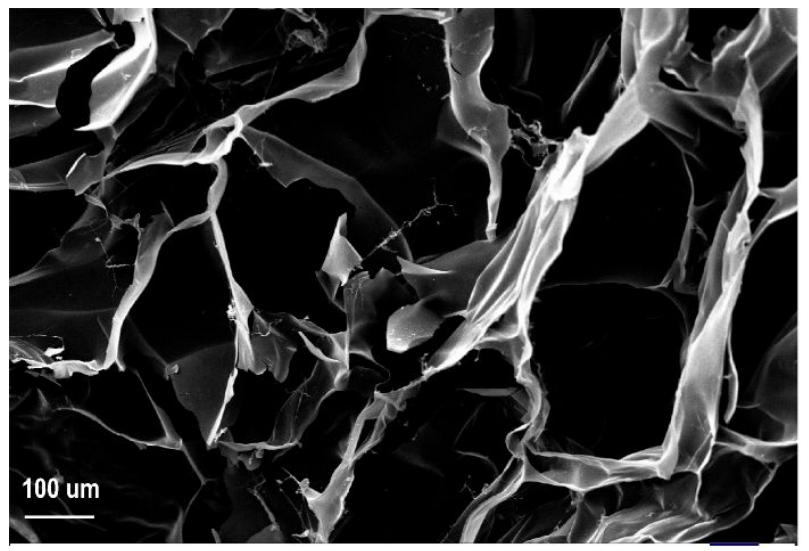

CIH8-0.6

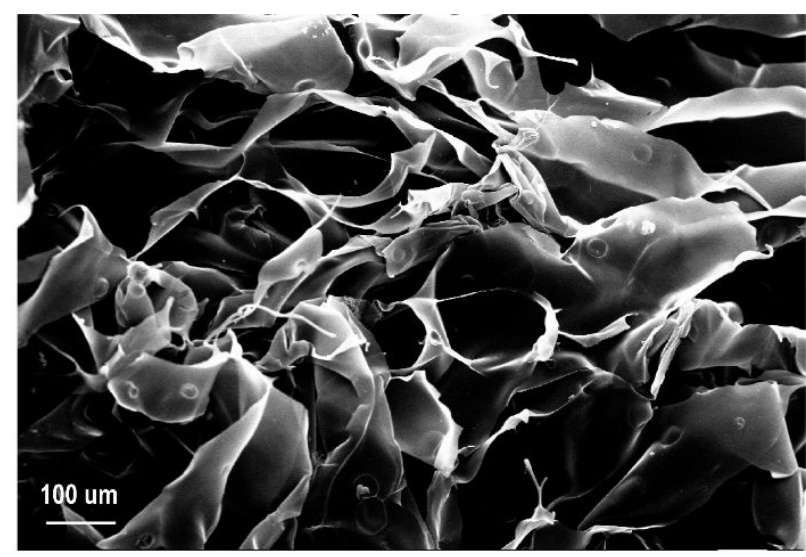

CIH8-2.0

Figure S3. Scanning electron microscopy (SEM) images of the CIH8-0.6 and CIH8-2.0. 
Table S2. Conditions and physical properties of CIHs

\begin{tabular}{|c|c|c|c|c|c|c|c|c|c|c|}
\hline \multirow[b]{2}{*}{ Samples } & \multirow[b]{2}{*}{ APS (\%) } & \multicolumn{4}{|c|}{ Compression } & \multicolumn{4}{|c|}{ Tension } & \multirow{2}{*}{$\begin{array}{c}\text { Swelling } \\
\text { ratios }\left(\mathrm{g} \mathrm{g}^{-1}\right)\end{array}$} \\
\hline & & $\begin{array}{l}\sigma_{\text {comp }}{ }^{a)} \\
(\mathrm{Kpa})\end{array}$ & $\begin{array}{c}\varepsilon_{\text {comp }}{ }^{b)} \\
(\%)\end{array}$ & $\begin{array}{l}\left.E_{\text {comp }}{ }^{c}\right) \\
(\mathrm{Kpa})\end{array}$ & $\begin{array}{c}\left.W_{f}^{d}\right) \\
\left(K J m^{-3}\right)\end{array}$ & $\begin{array}{l}\left.\sigma_{\text {Tens }}{ }^{\mathrm{e}}\right) \\
(\mathrm{Kpa})\end{array}$ & $\begin{array}{c}\varepsilon_{\text {Tens }}{ }^{f)} \\
(\%)\end{array}$ & $\begin{array}{l}\left.\mathrm{E}_{\mathrm{Tens}} \mathrm{g}\right) \\
(\mathrm{Kpa})\end{array}$ & $\begin{array}{c}W_{f}^{\mathrm{h})} \\
\left(\mathrm{KJ} \mathrm{m}^{-3}\right)\end{array}$ & \\
\hline CIH8-0.6 & 0.6 & 200 & 78.67 & 1.7 & 13.0 & 4.9 & 107 & 5 & 2.02 & 66.09 \\
\hline CIH8-1.0 & 1.0 & 290 & 80.05 & 3.6 & 17.20 & 16 & 126 & 12 & 6.20 & 47.95 \\
\hline CIH8-1.5 & 1.5 & 390 & 72.10 & 9.4 & 21.80 & 37 & 120 & 5.8 & 11.30 & 41.34 \\
\hline CIH8-2.0 & 2.0 & 480 & 71.61 & 14 & 28.20 & 49 & 129 & 12 & 19.50 & 35.50 \\
\hline ClH8-2.5 & 2.5 & 360 & 69.49 & 21 & 23.40 & 36 & 96 & 13 & 11.0 & 29.86 \\
\hline CIH8-3.0 & 3.0 & 220 & 61.13 & 36 & 16.20 & 36 & 80 & 14 & 9.10 & 26.82 \\
\hline CIH6-2.0 & 2.0 & 120 & 75.18 & 2.7 & 8.60 & 7.9 & 82 & 15 & 2.83 & 79.38 \\
\hline CIH7-2.0 & 2.0 & 490 & 78.72 & 5.5 & 29.60 & 15 & 126 & 12 & 6.17 & 61.68 \\
\hline ClH9-2.0 & 2.0 & 350 & 62.04 & 40 & 22.60 & 39 & 103 & 21 & 15.17 & 23.18 \\
\hline
\end{tabular}

a), b), c), and d) Stress at fracture, fracture strain, modulus, and work of fracture under compression; e), f), g), and h) tensile strength, elongation at break, Young's modulus, and work of fracture under tension, respectively.

Table S3. Summary of compression properties of pure cellulose based hydrogels reported in literatures

\begin{tabular}{|c|c|c|c|c|}
\hline & \multirow{2}{*}{ Materials } & \multicolumn{2}{|c|}{ Compression } & \multirow{2}{*}{ Ref } \\
\hline & & $\varepsilon_{\text {comp }}(\%)$ & $\sigma_{\text {comp }}(\mathrm{MPa})$ & \\
\hline \multirow{3}{*}{ Chemical cross-linking } & Microcrystalline cellulose (MCC) & 80.0 & 1.53 & 2 \\
\hline & Quaternized cellulose/ cellulose & $\sim 28.0$ & 0.031 & 3 \\
\hline & Allyl cellulose & 80.10 & 0.29 & This study \\
\hline \multirow{4}{*}{ Physical cross-linking } & Xylan/ cellulose nanocrystals (CNC) & 50.0 & 0.11 & 4 \\
\hline & Cellulose & $\sim 70.0$ & $\sim 3.0$ & 5 \\
\hline & Cellulose/ CNC & $\sim 55.0$ & $\sim 2.80$ & 5 \\
\hline & Dialdehyde microfibrillated cellulose/ gelatin & 78.0 & 1.70 & 6 \\
\hline Double-cross-linking & Cellulose & 74.0 & 4.80 & 7 \\
\hline
\end{tabular}


Table S4. Summary of tensile properties of pure natural polymer-based hydrogels including cellulose, chitin, and chitosan reported in literatures

\begin{tabular}{|c|c|c|c|c|}
\hline & \multirow{2}{*}{ Materials } & \multicolumn{2}{|c|}{ Tension } & \multirow{2}{*}{ Ref } \\
\hline & & $\varepsilon_{\text {Tens }}(\%)$ & $\sigma_{\text {Tens }}(\mathrm{MPa})$ & \\
\hline \multirow{3}{*}{ Chemical cross-linking } & Chitosan/ carrageenan & 109.74 & 0.53 & 8 \\
\hline & Chitosan & 117.30 & 0.37 & 9 \\
\hline & Allyl cellulose & 123.0 & 0.016 & This study \\
\hline \multirow{4}{*}{ Physical cross-linking } & Chitosan & 83.90 & 0.21 & 9 \\
\hline & Cellulose & 66.0 & 3.70 & 10 \\
\hline & Cellulose nanofiber & 21.0 & 5.40 & 11 \\
\hline & Chitin nanofiber & 52.20 & 7.0 & 12 \\
\hline Double-cross-linking & Cellulose & 86.0 & 1.60 & 7 \\
\hline
\end{tabular}



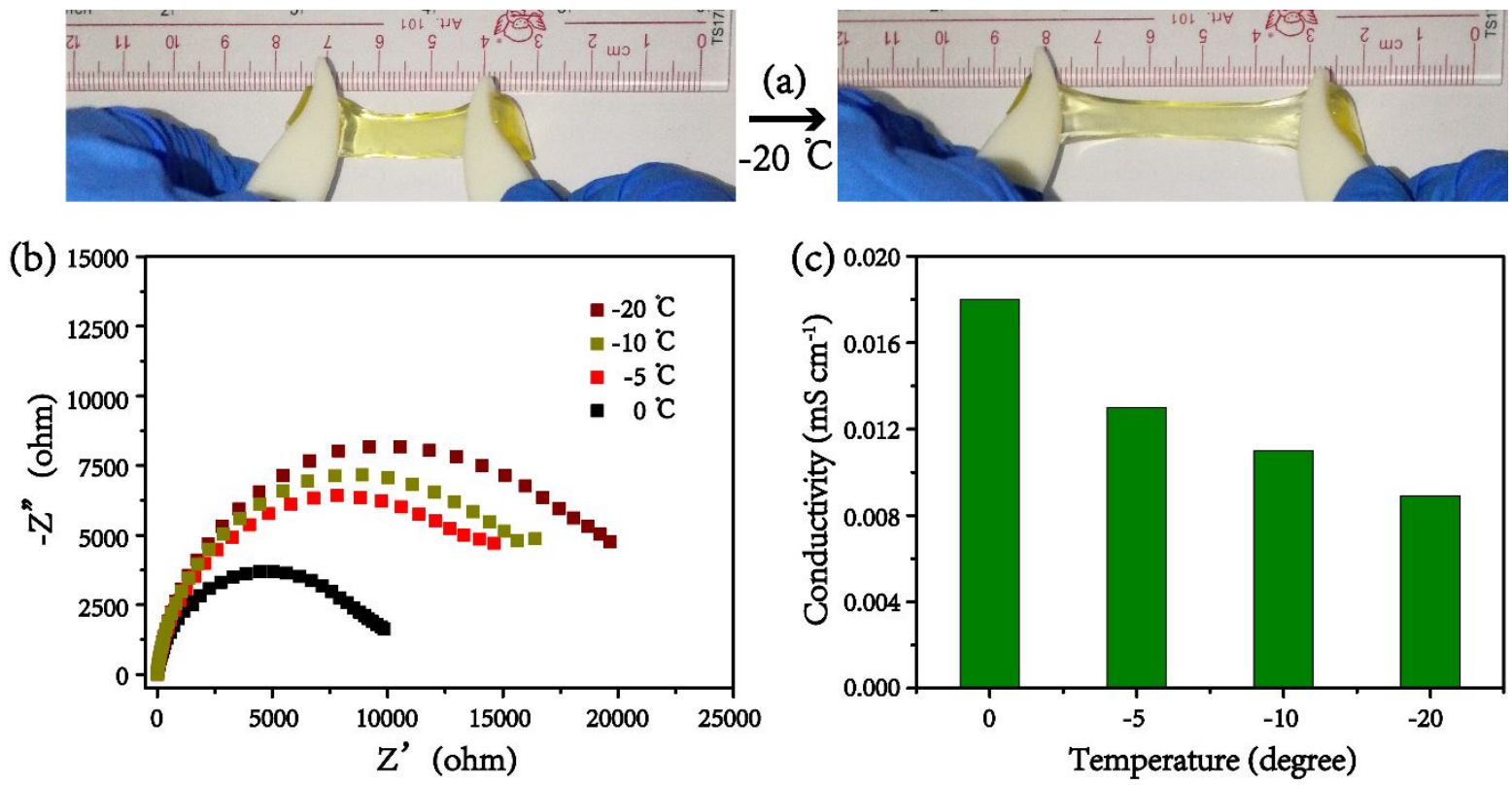

Figure S4. (a) Digital image of a uniaxial force stretched CIH8-1.0 at $-20{ }^{\circ} \mathrm{C}$. (b) Electrochemical impedance spectroscopy plots of CIH8-1.0, and (c) the calculated conductivity values for CIH8-1.0 at various low temperatures. The subzero experiments were carry out in the refrigerator. 


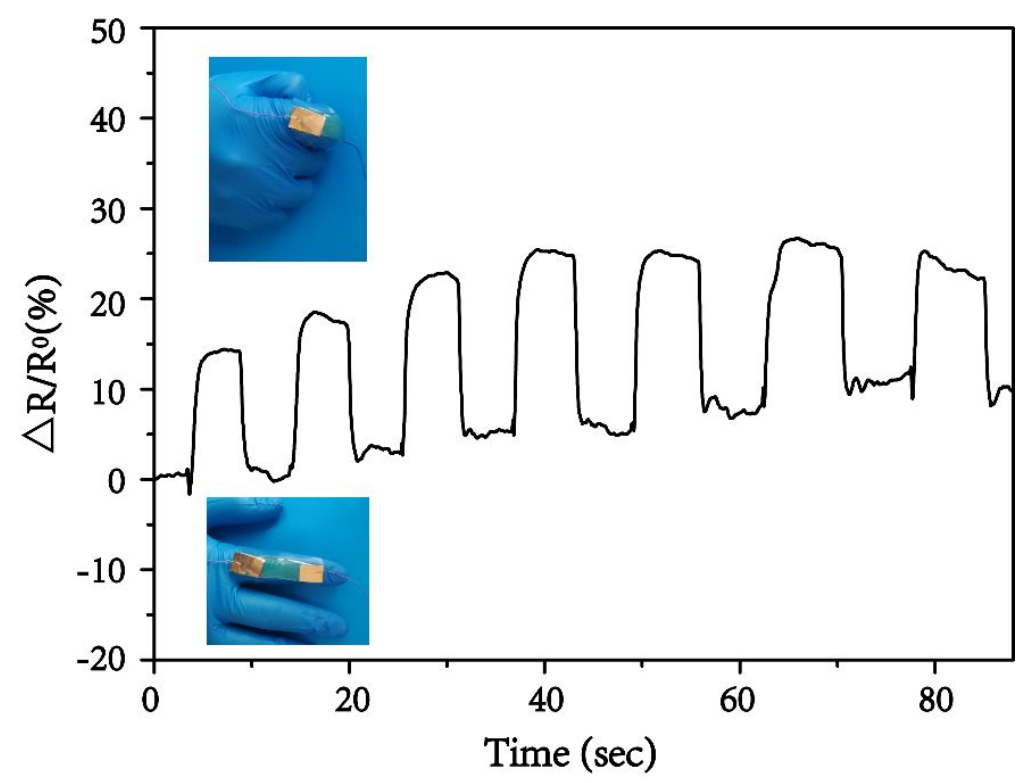

Figure S5. Response of CIH as strain sensor in monitoring human activities of finger bending.

(1) Hachet, E.; Sereni, N.; Pignot-Paintrand, I.; Ravaine, V.; Szarpak-Jankowska, A.; Auzely-Velty, R. Thiol-ene Clickable Hyaluronans: from Macro-to Nanogels. J. Colloid. Interf. Sci. 2014, 419, 52-55.

(2) Liang, X.; Qu, B.; Li, J.; Xiao, H.; He, B.; Qian, L. Preparation of Cellulose-Based Conductive Hydrogels with Ionic Liquid. React. Funct. Polym. 2015, 86, 1-6.

(3) Peng, N.; Wang, Y.; Ye, Q.; Liang, L.; An, Y.; Li, Q.; Chang, C. Biocompatible Cellulose-Based Superabsorbent Hydrogels with Antimicrobial Activity. Carbohydr. Polym. 2016, 137, 59-64.

(4) Kohnke, T.; Elder, T.; Theliander, H.; Ragauskas, A. J. Ice Templated and Cross-Linked Xylan/Nanocrystalline Cellulose Hydrogels. Carbohydr. Polym. 2014, 100, 24-30.

(5) Gao, X.; Sadasivuni, K. K.; Kim, H.-C.; Min, S.-K.; Kim, J. Designing pH-Responsive and Dielectric Hydrogels from Cellulose Nanocrystals. J. Chem. Sci. 2015, 127 (6), 1119-1125.

(6) Zheng, X.; Zhang, Q.; Liu, J.; Pei, Y.; Tang, K. A Unique High Mechanical Strength Dialdehyde Microfibrillated Cellulose/Gelatin Composite Hydrogel with a Giant Network Structure. RSC Adv. 2016, 6 (76), 71999-72007.

(7) Zhao, D.; Huang, J.; Zhong, Y.; Li, K.; Zhang, L.; Cai, J. High-Strength and High-Toughness Double-Cross-Linked Cellulose Hydrogels: A New Strategy Using Sequential Chemical and Physical Cross-Linking. Adv. Funct. Mater. 2016, 26 (34), $6279-6287$.

(8) Liang, X.; Wang, X.; Xu, Q.; Lu, Y.; Zhang, Y.; Xia, H.; Lu, A.; Zhang, L. Rubbery Chitosan/Carrageenan Hydrogels Constructed through an Electroneutrality System and Their Potential Application as Cartilage Scaffolds. Biomacromolecules 2018, 19 (2), $340-352$.

(9) Duan, J.; Liang, X.; Cao, Y.; Wang, S.; Zhang, L. High Strength Chitosan Hydrogels with Biocompatibility via New Avenue Based on Constructing Nanofibrous Architecture. Macromolecules 2015, 48 (8), 2706-2714.

(10) Shi, Z.; Gao, H.; Feng, J.; Ding, B.; Cao, X.; Kuga, S.; Wang, Y.; Zhang, L.; Cai, J. In Situ Synthesis of Robust Conductive Cellulose/Polypyrrole Composite Aerogels and Their Potential Application in Nerve Regeneration. Angew. Chem. Int. Ed. 2014, 53 (21), 5380-5384.

(11) Abe, K.; Yano, H. Cellulose Nanofiber-Based Hydrogels with High Mechanical Strength. Cellulose 2012, 19 (6), $1907-1912$.

(12) Abe, K.; Ifuku, S.; Kawata, M.; Yano, H. Preparation of Tough Hydrogels Based on $\beta$-Chitin Nanofibers via NaOH Treatment.

Cellulose 2013, 21 (1), 535-540. 